\title{
Maxillofacial Radiology 181
}

SADJ June 2020, Vol. 75 No. 5 p271

CJ Nortjé

Below are images of two patients that presented at the department. The one patient was accidentally shot by a friend using a pellet gun (Fig.1 \& 2), while the other patient presented with a swelling of three month duration on the left side of the mandible (Fig. 3, 4, 5, $6 \&$ 7). Studying the images presented below what are the important lessons to learn regarding the final diagnosis of the two cases?
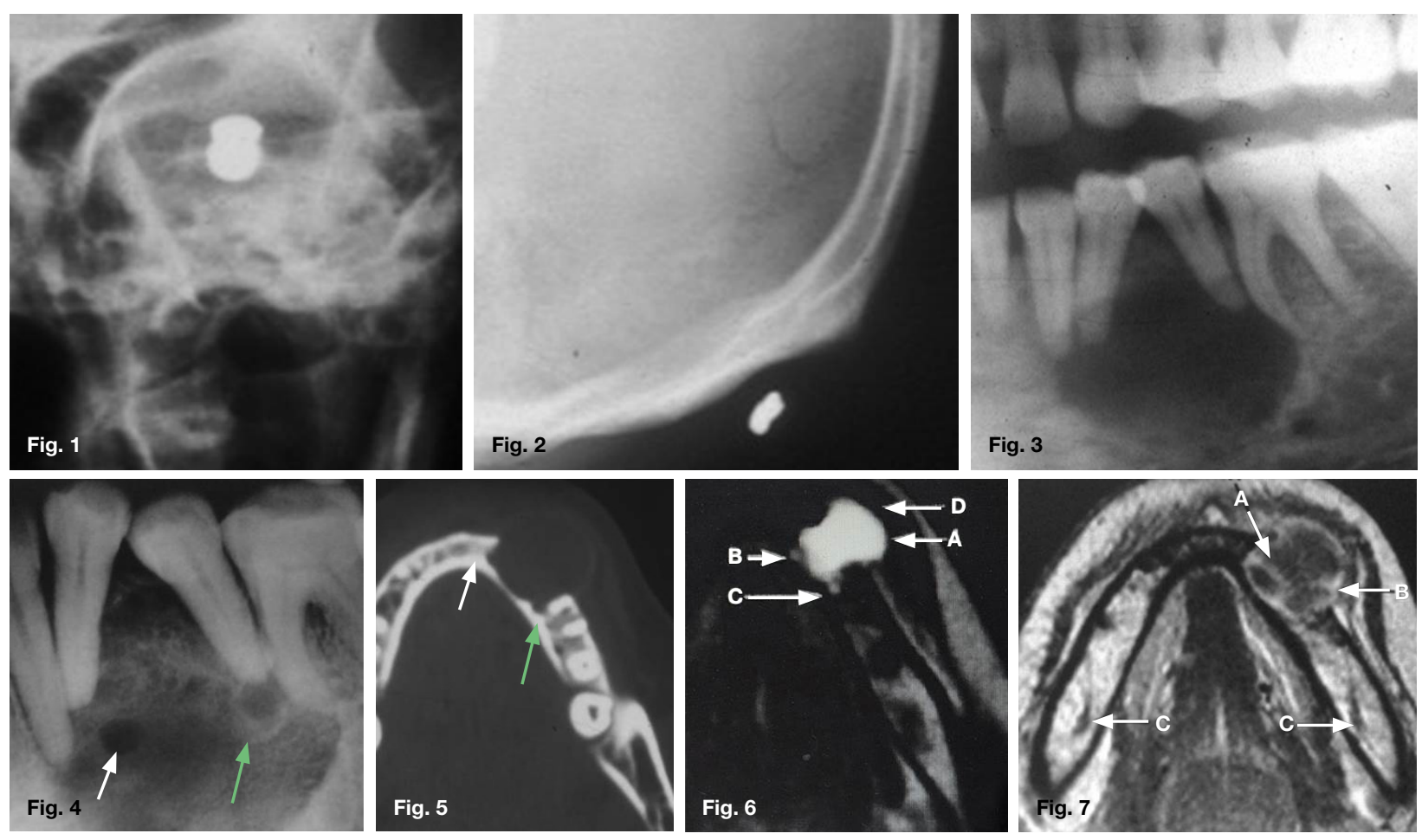

\section{INTERPRETATION}

A pantomograph was taken of the patient who was accidentally shot. No sign of the presence of a pellet was discernible on the pantomograph. A posterior-anterior skull radiograph (Fig. 1) was taken and the presence of a pellet in the eyeball are clearly demonstrated. The main reason why the pellet could not be observed on the pantomograph is probably due to the fact that the pellet was not in the layer when the radiograph was taken (Review Maxillofacial Radiology Case 180). Due to the presence of the pellet in the region of the eyeball, a lateral skull radiograph (Fig. 2) was taken which clearly show the presence of the pellet in the posterior region of the skull. "One view is no view" is a common phrase amongst radiologists. When conventional radiographs are used both AP and lateral radiographs are required before an accurate diagnosis can be made. A number of radiologists over the years got themselves in serious allegations of misconception due to relying on one view only before making a diagnosis. A cropped pantomograph (Fig. 3) of the patient presenting with a swelling on the left side of the mandible showing a well demarcated radiolucency causing divergence and resorption of the roots of 34 and 35. An intraoral radiograph (Fig. 4) which

Christoffel J Nortjé: $B C h D, P h D, A B O M R, D S c$. Faculty of Dentistry, University of the Western Cape.

ORCID Number: 0000-0002-9717-5514

Email: cnortje@uwc.ac.za shows a well demarcated radiolucency with two smaller well defined lucencies (arrows) suggestive of daughter cysts within the lesion. The reason why the daughter cysts are nicely demonstrated on the intra-oral film is because the film is very close to the lesion while on the pantomograph the cysts are not depicted because they are not present in the layer. An axial CT bone window setting (Fig. 5) shows two outward projecting daughter cysts in profile. A T2-weighted axial MR image (Fig. 6) shows the dominant cyst (A) which appears homogeneous with hyperintense fluid and the anteriorly (B) and posteriorly (C) situated daughter cysts are well depicted. A T1-weighted axial MR image (Fig. 7) confirming the lesion with buccal expansion. There are contrast enhancing soft tissue septae (A) dividing the lesion into a multilocular lesion. A small soft tissue contrast enhancing nodule is also discernible (B). The inferior alveolar nerves are also clearly depicted (C). A final histological diagnosis of a unicystic amelolastoma was made. The purpose of this communication was to emphasise the important role imaging plays in the diagnosis of lesions of the maxillofacial region.

\section{Reference}

1. The application of MR and CT Imaging in the Diagnosis and Management of Maxillofacial tumours. LJ van Rensburg, DSc Thesis, Maxillofacial Radiology, University of the Western Cape. 